\title{
Review Article \\ The Role of Stress Regulation on Neural Plasticity in Pain Chronification
}

\author{
Xiaoyun $\mathrm{Li}^{1}$ and $\mathrm{Li} \mathrm{Hu}^{1,2}$ \\ ${ }^{1}$ Key Laboratory of Cognition and Personality, Ministry of Education and Faculty of Psychology, \\ Southwest University, Chongqing, China \\ ${ }^{2}$ CAS Key Laboratory of Mental Health, Institute of Psychology, Beijing, China \\ Correspondence should be addressed to Li Hu; huli@psych.ac.cn
}

Received 27 July 2016; Revised 2 November 2016; Accepted 14 November 2016

Academic Editor: Jason Huang

Copyright (C) $2016 \mathrm{X}$. Li and L. Hu. This is an open access article distributed under the Creative Commons Attribution License, which permits unrestricted use, distribution, and reproduction in any medium, provided the original work is properly cited.

\begin{abstract}
Pain, especially chronic pain, is one of the most common clinical symptoms and has been considered as a worldwide healthcare problem. The transition from acute to chronic pain is accompanied by a chain of alterations in physiology, pathology, and psychology. Increasing clinical studies and complementary animal models have elucidated effects of stress regulation on the pain chronification via investigating activations of the hypothalamic-pituitary-adrenal (HPA) axis and changes in some crucial brain regions, including the amygdala, prefrontal cortex, and hippocampus. Although individuals suffer from acute pain benefit from such physiological alterations, chronic pain is commonly associated with maladaptive responses, like the HPA dysfunction and abnormal brain plasticity. However, the causal relationship among pain chronification, stress regulation, and brain alterations is rarely discussed. To call for more attention on this issue, we review recent findings obtained from clinical populations and animal models, propose an integrated stress model of pain chronification based on the existing models in perspectives of environmental influences and genetic predispositions, and discuss the significance of investigating the role of stress regulation on brain alteration in pain chronification for various clinical applications.
\end{abstract}

\section{Introduction}

Chronic pain is a main source of worldwide disability, causing physical and psychological discomforts and rising huge medical expenses [1]. Understanding mechanisms of the development of chronic pain is crucial in monitoring and preventing the progress of pain chronification. In recent decades, increasing clinical studies and complementary animal models contributed to important advances in understanding the transition from acute to chronic pain. Notably, Melzack [2] proposed that stress played an important role in such pain chronification, and accumulating evidence demonstrated that stress regulation (as indexed by the function of hypothalamic-pituitary-adrenal [HPA] axis) consistently engaged in the development of chronic pain [3-5]. In line with these findings, several brain regions, subserving as key candidates for stress regulation [6-8], have been reported to be involved in the transition from acute to chronic pain, including the amygdala, prefrontal cortex (PFC), and hippocampus [9-12]. Therefore, some previous studies hypothesized that these brain regions, especially within the emotional corticolimbic system, acted as the bridge of pain modulation and stress regulation. In this paper, we briefly walk through concepts of pain and stress, review effects of the HPA function on acute and chronic pain, and discuss alterations of stressassociated brain regions in acute and chronic pain. In the following, we discuss two existing stress models of chronic pain in perspectives of environmental influences and genetic predispositions, respectively, and propose an integrated stress model of pain chronification based on previous findings.

\section{Acute Pain and Chronic Pain}

Pain is a conscious sensation, processing multidimensional information involving sensory, affective, and cognitive components [2]. Acute pain, serving as a warning of injury or 
illness, functions to protect the organs from a present or potential damage. If acute pain continues for a long time (e.g., longer than 3 months), it can develop into chronic pain, even after the initial injury or illness has been healed [13]. In addition, the chronic pain itself is usually associated with hyperalgesia and/or allodynia [14] and psychological distress, such as anxiety and depression [15].

\section{Stress and the HPA Axis}

Stress is a biological reaction [2] that triggers a rapid response by activating the sympathetic nervous system and a relative slower response via evoking the HPA axis [8]. The faster pathway releases catecholamines, such as adrenaline and noradrenaline, priming the body into a classic "fight or flight" mode with enhanced activation of the sympathetic nervous system (e.g., increased heart rate and blood pressure, sweat gland activation). When the slower stress response pathway of the HPA axis is activated, corticotropin-releasing factor (CRF) travels from the paraventricular nucleus (PVN) of the hypothalamus to the pituitary, leading to the release of adrenocorticotropic hormone (ACTH). In turn, ACTH stimulates the adrenal gland to secrete glucocorticoids (cortisol in humans, corticosterone in rodents) that are essential for stress response. This type of hormone can naturally go through the blood-brain barrier and access multiple brain regions, like the amygdala, PFC, and hippocampus, binding with two intracellular receptors, the glucocorticoid receptor and the mineralocorticoid receptor [16]. As such, glucocorticoids can influence neuronal excitability and synaptic and neuronal plasticity $[17,18]$.

\section{The Effect of Stress Regulation on Pain}

Studies associated with stress responses have demonstrated that altered activations of the HPA axis are in response to experimental pain $[19,20]$ and various chronic pain disorders $[3,5]$. Conceptually, pain-related activation of the HPA axis has been embedded in an allostatic load model of disease $[4,21]$. This model assumes that, in response to acute stress, various physiological activities could be activated to help organism adapt to environmental changes. It has also been proposed that acute pain induced cortisol elevation may reduce pain unpleasantness [22] and increase pain tolerance [23], which provides solid evidence for the transient stressinduced analgesia [24]. However, when stress, induced by physical injury and/or pain-related psychological factors, is prolonged, uncertain, and uncontrollable, the response of such stress becomes maladaptive, entering into a vicious cycle, in which acute pain has evolved into a chronic state with abnormal alterations of brain structures and functions [4, 21]. Meanwhile, cortisol fails to act its protective functions under chronic pain conditions, thereby exaggerating pain severity. In fact, emerging evidence suggests that chronic pain is associated with the dysfunction of cortisol secretion, although the nature of their relationship is not fully elucidated. A majority of previous studies reported reduced cortisol secretions or lower basal levels of cortisol in various chronic pain disorders, such as fibromyalgia $[25,26]$, chronic whiplash-associated disorder [27], chronic neck pain [28], chronic low back pain [29], chronic fatigue syndrome [30], and chronic pelvic pain [31]. Since the HPA axis is a self-regulating negative feedback system [7], such hypocortisolism is indicative of attenuated activity or impaired feedback sensitivity of the HPA axis. However, a few studies found elevated cortisol levels [3235], abnormal cortisol diurnal variations [36], and increased feedback sensitivity of the HPA axis [37] in certain chronic pain conditions, like fibromyalgia and chronic back pain. In contrast, several studies reported that the profiles of cortisol secretion in patients suffering with fibromyalgia [38, 39], chronic back pain $[40,41]$, or chronic temporomandibular disorders [42] did not differ from those in healthy controls. One possible explanation to these conflicting findings is that the HPA axis may be in the state of hyperactivity at the early stage of pain chronification, while, after long-term overt activity, the stress system reaches an exhausted state, thereby turning into the HPA axis hypoactivity [26]. Albeit such explanation requires further verification, it is reasonable to suggest that the HPA axis is involved in the development of chronic pain.

\section{Neural Plasticity in Acute and Chronic Pain}

The corticolimbic system, including amygdala, PFC, and hippocampus, is a powerful neural network that has been suggested to contribute to the transition from acute to chronic pain [12]. Further, both acute and chronic pain profoundly influence this system, which is also known to relate to stress regulation [8], via structural and functional alterations in the related brain regions. Compelling evidence supporting this conclusion was well documented by various human neuroimaging studies and animal models.

5.1. Brain Responses to Acute Pain. Most human neuroimaging studies on acute pain have revealed consistent activations in the insula and dorsal anterior cingulate cortex [43-45]. Even less consistency, activations in the amygdala, PFC, and hippocampus have been frequently reported in neuroimaging studies of acute pain [46-51]. Specifically, noxious stimulation applied on pain-free individuals evoked stronger Blood-Oxygen-Level-Dependent signals in the PFC [46, 5153] and hippocampus [46, 48, 54, 55]. Besides, activations of amygdala and hippocampus were observed to be related to pain expectancy $[53,55,56]$. Furthermore, augmented activation of amygdala was induced by pain in depressed individuals [57] and in healthy cohort with greater pain unpleasantness followed by an induction of depressive mood [52], suggesting the role of amygdala in the integration of pain and emotional information. In addition, the HPA axis influences the activations of the abovementioned brain regions under acute pain conditions. For instance, previous studies demonstrated that elevated cortisol levels were (1) associated with reduced pain unpleasantness and decreased pain-related brain activation during constant noxious stimulation [22], (2) linked with lower pain threshold and stronger PFC activity in response to inflammation-induced pain [58], and (3) related 
to enhanced hippocampal activation during step-up noxious stimulation (an increasing pattern of noxious stimulation) [59]. These studies suggested that acute pain, acting in a similar way with acute stress, may evoke cortisol levels to boost the survival of the organism by inhibiting and/or facilitating activities of related brain networks. Although no path analysis in these studies was performed to verify the interrelationship among acute pain, stress regulation, and functional changes of the corticolimbic system, it is highly likely that acute pain may evoke an adaptive response to protect the organism via the coinfluence of cortisol elevation and brain responses in the amygdala, PFC, and hippocampus.

In fact, evidence from animal models suggests that this may be the same case. Consistent observations of acute pain-related functional changes in the corticolimbic system have been reported in animal studies. For instance, noxious stimulation induced neuronal excitability in the amygdala [60-62] and PFC [63] and neuronal inhibition in the CA1 hippocampus $[64,65]$. In line with the electrophysiological findings, immunohistochemical and fMRI studies also showed similar activation patterns in these brain regions by delivering noxious stimulation on rats [63, 66-69]. These findings confirmed the role of acute pain on brain plasticity. Additionally, it is important to find out the role of stress regulation in the changes of brain responses under acute pain conditions, and evidence from an immunocytochemical study might shed light on this issue [70]. In this study, rats with less pain sensitivity showed less freezing responses, stronger vocalization, and increased PFC activation and plasma corticosterone levels, in response to conditioned aversive stimuli. The authors also found an increased Fos expression (an indicator of neuronal activity in rats) in the hypothalamus and dentate gyrus of the hippocampus in the same group of rats. In contrast, rats with high pain sensitivity responded more passively to aversive events (e.g., more freezing behavior and weaker vocalization), along with increased activation in the amygdala and CA1 hippocampus, but did not show significant changes in corticosterone levels [70]. These findings suggested that enhanced activity of stress axis in rats with low pain sensitivity was due to the role of the PFC and hippocampus in regulation of glucocorticoid release, which was crucial for survival, while such regulation was ineffective in rats with high pain sensitivity [70].

In summary, human and animal studies of acute pain have provided evidence in support of the viewpoint that acute pain, similar to acute stress, increased the release of glucocorticoid. Meanwhile, the above discussed brain regions are highly sensitive to acute painful and stressful stimuli and are highly plastic via regulation of the glucocorticoid negative feedback in the PFC and hippocampus. Therefore, it is conceivable that the whole process can be considered as the reaction in an adaptive and protective response system to aversive stimuli, leading to a better adaptation for survival.

\subsection{Brain Alterations in Chronic Pain. Human neuroimaging} studies have emphasized the association between chronic pain and abnormal changes in gray matter volume and thickness of the brain in various chronic pain patients [71-73]. Remarkably, a large number of studies have repeatedly showed reduced volumes of the amygdala $[12,74-78]$, medial prefrontal cortex (mPFC) [71, 74, 79-81], and hippocampus $[32,71,81-83]$ in a variety of chronic pain populations. In accordance with the morphological alterations, functional changes of the amygdala, $\mathrm{mPFC}$, and hippocampus were also observed under chronic pain conditions [47, 84, 85]. A metaanalysis study reported that peak activation was found in the basolateral amygdala in clinical populations, suggesting the enhanced cognitive-affective processing among patients suffering from chronic pain [85]. In chronic pain patients, clinical pain intensity appeared to be associated with greater activations in the mPFC $[86,87]$ and hippocampus [32]. Additionally, under the circumstance of clinical pain fluctuation, greater functional connectivity of mPFC with the limbic system, including the amygdala and hippocampus, was indicative of pain chronification $[9,10,12,85,88]$. Although stress regulation and pain chronification are suggested to share a similar mechanism in modulating brain structures and functions [2], how stress regulation plays a role in brain alterations in patients with chronic pain remains unclear. Recently, a clinical study explored the influence of maladaptive stress response on pain state of patients, such as elevated cortisol levels that were associated with enhanced clinical pain intensity, smaller hippocampal volume, and stronger hippocampal activations [32], which provided strong evidence to demonstrate the important role of the maladaptive stress response on the transition from acute to chronic pain [4]. Interestingly, a recent study of chronic myofascial pain reported that gray matter atrophy in the PFC and hippocampus was independent of the cortisol levels. They also reported that only the gray matter density of PFC was negatively correlated with pain thresholds in patients, suggesting the presence of pain disinhibition [89]. Despite these findings are contradictive, it can be imaginable that dysfunction of the HPA axis and abnormal brain alterations may be caused by maladaptive responses to chronic pain.

In support of the above viewpoints, evidence from animal studies has unraveled the role of chronic pain in altering the structure and function of the corticolimbic system. The longlasting sympathic pain increased neuronal excitability and dendritic branching of amygdala in rodents [90, 91], resulting in an enlarged amygdala volume [92]. Spared nerve injury sympathic pain induced alterations of dendritic length, spine density, and neuronal activity in the $\operatorname{mPFC}[93,94]$, and longterm neuropathic pain reduced prefrontal volumes in rodents [95]. Moreover, hippocampal neurogenesis appeared to be suppressed in a rat model of neuropathic pain, subserving a possible mechanism of pain chronification $[83,96]$. Additionally, since the $\mathrm{mPFC}$ receives inputs from both amygdala and hippocampus [97], pain-related amygdala hyperactivity inhibited the mPFC activation and impaired functions related to decision-making in an arthritis pain model [98, 99], whereas reduced hippocampus-prefrontal connectivity was associated with impaired spatial memory performance in rats with neuropathic pain [100]. Yet the knowledge of influence of stress regulation on brain alterations in chronic pain is very limited. An animal study of chronic neuropathic pain reported that enhanced nociceptive sensitivity during chronic pain was associated with increased activation in the 
amygdala and decreased activation in the hippocampus but failed to influence activation of the HPA axis [101]. Interestingly, such dissociation between pain sensitivity and stress response in rats implicated that the HPA axis dysfunction in chronic pain patients might not originate from pain itself, but rather from other factors associated with repeated painful stimuli (e.g., experimental cues and an inability to escape from pain).

In summary, human neuroimaging studies and animal models of chronic pain have suggested that chronic pain could induce a chronic stress-like alteration in the HPA axis and the corticolimbic system, with dysregulation of the HPA axis, and dysfunction and reorganization of the corticolimbic system. It is reasonable to conclude that such maladaptive responses are likely to contribute to the development of chronic pain.

\section{Stress Models of Pain Chronification}

Given that activation of the HPA axis influences neural alterations, both structurally and functionally, the role of stress regulation becomes critical in the understanding of brain mechanisms in the evolution of acute pain into chronic pain.

From the perspective of stress regulation, human and animal studies suggest that chronic stress has an enduring and destructive effect on the brain via activation of the HPA axis, particularly the glucocorticoid secretion [102]. To explain this phenomenon, two different hypotheses have been proposed: the neurotoxicity hypothesis and the vulnerability hypothesis. The neurotoxicity hypothesis suggests that prolonged release of glucocorticoid impairs the neuronal capacity to resist toxic invasion or normal attrition, leading to the reduction of hippocampal volume in populations with chronic stress, including posttraumatic stress disorder (PTSD) and depression [103]. In contrast, the vulnerability hypothesis points out that small hippocampal volume is a predetermine risk factor for chronic stress that is shaped by genetic predispositions and/or early life stress [104], as evidenced in the studies of stress [105], anxiety [106], and PTSD [104].

Accordingly, two stress models of chronic pain have also been proposed, from environmental influence and genetic predisposition perspectives, respectively: a "neurotoxic model" and a "vulnerability model." The "neurotoxic model" suggests that persistent pain may lead to the maladaptive stress response, such as dysfunction of the HPA axis, affecting the alterations in brain structure and function $[6,107]$. In line with this model, chronic pain-related changes in the corticolimbic system, including the amygdala, PFC, and hippocampus $[47,71,73,108]$, have been considered as a consequence of allostatic load of chronic disease, in which prolonged pain dysregulates the HPA axis, thereby impairing brain structures and functions [4]. In contrast, the "vulnerability model" proposes that the characteristics of some particular brain structures, such as the volume of the brain regions within the corticolimbic system [109], may contribute to the vulnerability of development from acute to chronic pain, thus affecting the activation of the HPA axis and brain functions $[105,110]$. For example, a longitudinal study tracked brain alterations for 3 years in patients with subacute back pain as they gradually evolved to chronic pain states and found that small sizes of amygdala and hippocampus were the preexisting risk factors in the development of chronic back pain [12].

It is of interest to note that a recent study of chronic back pain using path analysis has suggested that small hippocampal volume has represented a risk factor of vulnerability to persist pain in a maladaptive stress response manner (the "vulnerability model") [32]. However, their observation cannot disprove the "neurotoxic model" because the development of chronic back pain, similar to other chronic pain diseases, is a dynamic process, and any cross-sectional study cannot track the causal relationship among pain chronification, stress regulation, and brain alterations during the whole process. Indeed, we are not able to rule out the possibility that the influence of stress response in pain chronification is driven by the combination of both environmental and genetic factors. It should be noted that emerging studies in the perspectives of stress regulation or pain chronification support this possibility $[102,111]$. We presume that the level of activation of the HPA axis is determined by the combination of vulnerable factors (e.g., structural characteristics of the corticolimbic system) and environmental factors (e.g., pain caused by an injury) and influences the alterations of the structure and function of the corticolimbic system. Subsequently, these physiological responses would lead to either the recovery of health or the persistence of pain state that in turn strengthens brain reorganizations and eventually develops into a chronic pain state (Figure 1). Besides, the proposed stress model of pain chronification should be verified in a longitudinal design by tracking the alterations of all physiological responses, including brain structures and functions, and glucocorticoid levels. We foresee that unraveling the stress effect on brain mechanisms in pain chronification will be of great importance to predict the development of chronic pain [9-12], thus having important clinical significance.

\section{Conclusion}

We have provided a broad range of compelling evidence that pain modulation and stress regulation can be considered as an integrated processing [2], within the framework of corticolimbic system. Human neuroimaging studies and animal models have unfolded a field of vision in the mechanism that drives the development of chronic pain under the HPA axis regulation. In contrast to the fact that activations of the HPA axis and the corticolimbic system show an adaptive manner to the perceived danger in acute pain, chronic pain is commonly associated with dysfunction of the HPA axis and brain reorganizations. Based on the two existing stress models of chronic pain, we propose an integrated stress model of pain chronification, emphasizing the integration of contingent environmental influences and genetic predispositions in the 


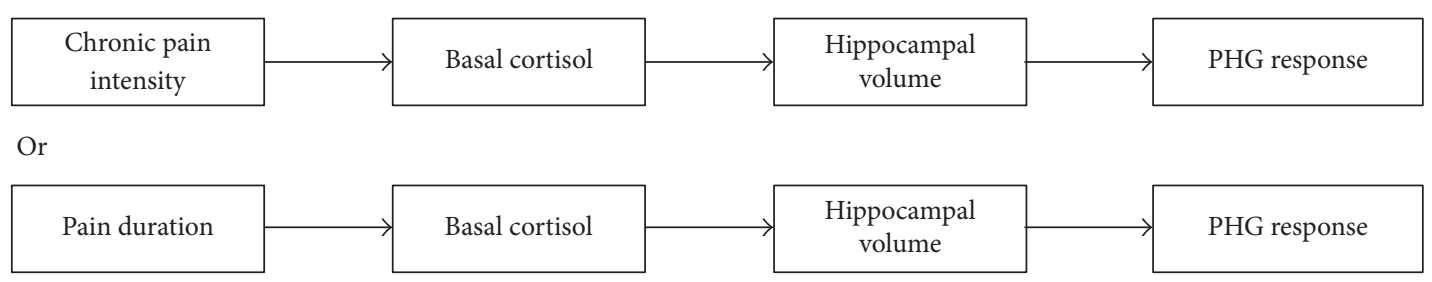

(a) Neurotoxic model

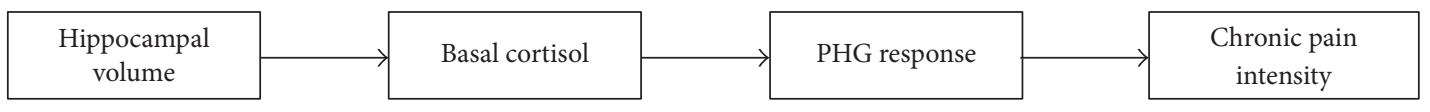

(b) Vulnerability model

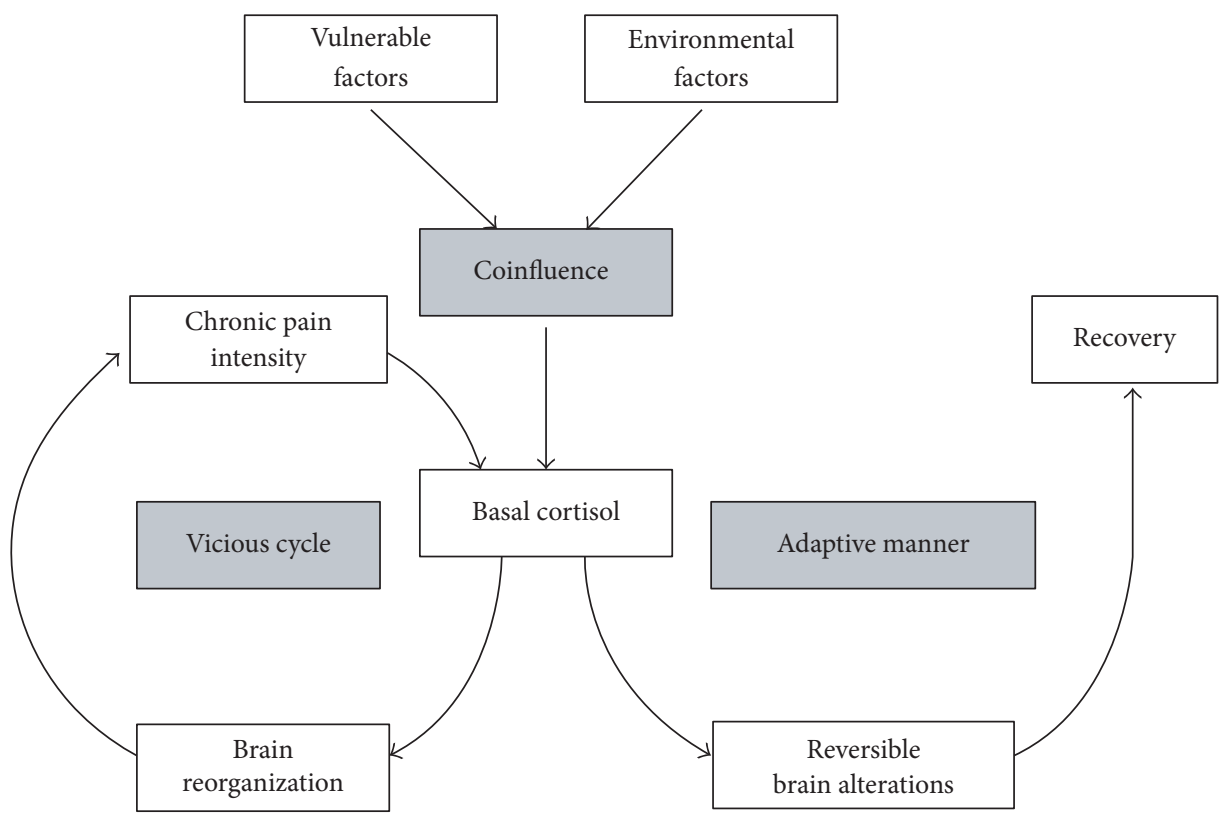

(c) Proposed model of pain chronification

FIGURE 1: Stress models of chronic pain. (a) The neurotoxic model, from the perspective of environmental influences, conceptualizes that chronic pain intensity or pain duration may lead to the maladaptive stress response, affecting the structure and function of the hippocampal formation and the parahippocampal gyrus (PHG) [32]. (b) The vulnerability model, from the perspective of genetic predispositions, conceptualizes that the small hippocampal volume as a vulnerable factor affects the levels of stress hormones, which in turn lead to enhanced activations of the parahippocampal gyrus and increased persistent pain intensity [32]. (c) The proposed integrated model, from the perspective of the combination of environmental influences and genetic predispositions, conceptualizes that vulnerable factors (e.g., properties of particular brain structures) and environmental factors (e.g., injury) codetermine the levels of basal cortisol, which result in the brain alterations. Thereafter, these physiological responses either return to normal levels with an adaptive manner or initiate a vicious cycle of cortisol dysfunction, brain reorganizations, and persistent pain state.

transition from acute to chronic pain. However, the precise mechanism of this integrated model remains to be investigated. Hopefully, future studies can determine physiological responses in relation to activation of the HPA axis and alterations of the corticolimbic system as the specific biomarkers of pain chronification, for monitoring and preventing the transition to chronic pain. The identification of these biomarkers in patients suffering from acute and chronic pain is important, as it can help facilitate the effective pain rehabilitation, reduce pain-related disability, and improve quality of life.

\section{Competing Interests}

The authors declare that there are no competing interests regarding the publication of this paper.

\section{Acknowledgments}

Li Hu is supported by the National Natural Science Foundation of China (nos. 31471082 and 31671141), Chongqing Research Program of Basic Research and Frontier Technology (no. cstc2015jcyjBX0050), and the Scientific Foundation 
Project of Institute of Psychology, Chinese Academy of Sciences (no. Y6CX021008).

\section{References}

[1] C. J. L. Murray and A. D. Lopez, "Measuring the global burden of disease," New England Journal of Medicine, vol. 369, no. 5, pp. 448-457, 2013.

[2] R. Melzack, "Pain and stress: a new perspective," in Psychological Factors in Pain, R. J. Gatchel and D. C. Turk, Eds., pp. 89-106, Guildford Press, New York, NY, USA, 1999.

[3] G. Blackburn-Munro and R. E. Blackburn-Munro, "Chronic pain, chronic stress and depression: coincidence or consequence?" Journal of Neuroendocrinology, vol. 13, no. 12, pp. 1009-1023, 2001.

[4] D. Borsook, N. Maleki, L. Becerra, and B. McEwen, "Understanding migraine through the lens of maladaptive stress responses: a model disease of allostatic load," Neuron, vol. 73, no. 2, pp. 219-234, 2012.

[5] B. S. McEwen and M. Kalia, "The role of corticosteroids and stress in chronic pain conditions," Metabolism: Clinical and Experimental, vol. 59, no. 1, p. -S15, 2010.

[6] E. R. de Kloet, M. Joëls, and F. Holsboer, "Stress and the brain: from adaptation to disease," Nature Reviews Neuroscience, vol. 6, no. 6, pp. 463-475, 2005.

[7] J. P. Herman, M. M. Ostrander, N. K. Mueller, and H. Figueiredo, "Limbic system mechanisms of stress regulation: hypothalamo-pituitary- adrenocortical axis," Progress in Neuro-Psychopharmacology and Biological Psychiatry, vol. 29, no. 8, pp. 12011213, 2005.

[8] B. S. McEwen, "Physiology and neurobiology of stress and adaptation: central role of the brain," Physiological Reviews, vol. 87, no. 3, pp. 873-904, 2007.

[9] M. N. Baliki, B. Petre, S. Torbey et al., "Corticostriatal functional connectivity predicts transition to chronic back pain," Nature Neuroscience, vol. 15, no. 8, pp. 1117-1119, 2012.

[10] J. A. Hashmi, M. N. Baliki, L. Huang et al., "Shape shifting pain: chronification of back pain shifts brain representation from nociceptive to emotional circuits," Brain, vol. 136, no. 9, pp. 2751-2768, 2013.

[11] A. R. Mansour, M. N. Baliki, L. Huang et al., "Brain white matter structural properties predict transition to chronic pain," Pain, vol. 154, no. 10, pp. 2160-2168, 2013.

[12] E. Vachon-Presseau, P. Tétreault, B. Petre et al., "Corticolimbic anatomical characteristics predetermine risk for chronic pain," Brain, vol. 139, no. 7, pp. 1958-1970, 2016.

[13] H. Merskey and N. Bogduk, Classification of Chronic Pain: Descriptions of Chronic Pain Syndromes and Definitions of Pain Terms, IASP Press, Seattle, Wash, USA, 2nd edition, 1994.

[14] M. J. Millan, "The induction of pain: an integrative review," Progress in Neurobiology, vol. 57, no. 1, pp. 1-164, 1999.

[15] S. J. Linton, "A review of psychological risk factors in back and neck pain," Spine, vol. 25, no. 9, pp. 1148-1156, 2000.

[16] M. Joëls, H. Karst, R. DeRijk, and E. R. de Kloet, "The coming out of the brain mineralocorticoid receptor," Trends in Neurosciences, vol. 31, no. 1, pp. 1-7, 2008.

[17] B. S. McEwen, "Plasticity of the hippocampus: adaptation to chronic stress and allostatic load," Annals of the New York Academy of Sciences, vol. 933, pp. 265-277, 2001.
[18] B. Roozendaal, B. S. McEwen, and S. Chattarji, "Stress, memory and the amygdala," Nature Reviews Neuroscience, vol. 10, no. 6, pp. 423-433, 2009.

[19] M. Al'Absi, K. L. Petersen, and L. E. Wittmers, "Adrenocortical and hemodynamic predictors of pain perception in men and women," Pain, vol. 96, no. 1-2, pp. 197-204, 2002.

[20] C. Zimmer, H.-D. Basler, H. Vedder, and S. Lautenbacher, "Sex differences in cortisol response to noxious stress," Clinical Journal of Pain, vol. 19, no. 4, pp. 233-239, 2003.

[21] B. S. McEwen, "Stress, adaptation, and disease: allostasis and allostatic load," Annals of the New York Academy of Sciences, vol. 840, pp. 33-44, 1998.

[22] E. Vachon-Presseau, M.-O. Martel, M. Roy et al., "Acute stress contributes to individual differences in pain and pain-related brain activity in healthy and chronic pain patients," The Journal of Neuroscience, vol. 33, no. 16, pp. 6826-6833, 2013.

[23] K. E. Dixon, B. E. Thorn, and L. C. Ward, "An evaluation of sex differences in psychological and physiological responses to experimentally-induced pain: a path analytic description," Pain, vol. 112, no. 1-2, pp. 188-196, 2004.

[24] N. I. Yarushkina, T. R. Bagaeva, and L. P. Filaretova, "Central corticotropin-releasing factor (CRF) may attenuate somatic pain sensitivity through involvement of glucocorticoids," Journal of Physiology \& Pharmacology, vol. 62, no. 5, pp. 541-548, 2011.

[25] L. J. Crofford, "The hypothalamic-pituitary-adrenal stress axis in fibromyalgia and chronic fatigue syndrome," Zeitschrift fur Rheumatologie, vol. 57, S2, pp. S67-S71, 1998.

[26] R. Riva, P. J. Mork, R. H. Westgaard, and U. Lundberg, “Comparison of the cortisol awakening response in women with shoulder and neck pain and women with fibromyalgia," Psychoneuroendocrinology, vol. 37, no. 2, pp. 299-306, 2012.

[27] J. Gaab, S. Baumann, A. Budnoik, H. Gmünder, N. Hottinger, and U. Ehlert, "Reduced reactivity and enhanced negative feedback sensitivity of the hypothalamus-pituitary-adrenal axis in chronic whiplash-associated disorder," Pain, vol. 119, no. 1-3, pp. 219-224, 2005.

[28] L. Karlsson, B. Gerdle, B. Ghafouri et al., "Intramuscular pain modulatory substances before and after exercise in women with chronic neck pain," European Journal of Pain, vol. 19, no. 8, pp. 1075-1085, 2015.

[29] C. Muhtz, R. Rodriguez-Raecke, K. Hinkelmann et al., "Cortisol Response to Experimental Pain in Patients with Chronic Low Back Pain and Patients with Major Depression," Pain Medicine (United States), vol. 14, no. 4, pp. 498-503, 2013.

[30] S. L. Nijhof, J. M. T. M. Rutten, C. S. P. M. Uiterwaal, G. Bleijenberg, J. L. L. Kimpen, and E. M. V. D. Putte, "The role of hypocortisolism in chronic fatigue syndrome," Psychoneuroendocrinology, vol. 42, pp. 199-206, 2014.

[31] K. F. S. Petrelluzzi, M. C. Garcia, C. A. Petta, D. M. GrassiKassisse, and R. C. Spadari-Bratfisch, "Salivary cortisol concentrations, stress and quality of life in women with endometriosis and chronic pelvic pain," Stress, vol. 11, no. 5, pp. 390-397, 2008.

[32] E. Vachon-Presseau, M. Roy, M.-O. Martel et al., "The stress model of chronic pain: evidence from basal cortisol and hippocampal structure and function in humans," Brain, vol. 136, no. 3, pp. 815-827, 2013.

[33] D. Catley, A. T. Kaell, C. Kirschbaum, and A. A. Stone, "A naturalistic evaluation of cortisol secretion in persons with fibromyalgia and rheumatoid arthritis," Arthritis Care \& Research, vol. 13, no. 1, pp. 51-61, 2000. 
[34] L. J. Crofford, S. R. Pillemer, K. T. Kalogeras et al., "Hypothalamic-pituitary-adrenal axis perturbations in patients with fibromyalgia," Arthritis \& Rheumatism, vol. 37, no. 11, pp. 1583-1592, 1994.

[35] G. Neeck and W. Riedel, "Hormonal pertubations in fibromyalgia syndrome," Annals of the New York Academy of Sciences, vol. 876, pp. 325-339, 1999.

[36] G. A. McCain and K. S. Tilbe, "Diurnal hormone variation in fibromyalgia syndrome: a comparison with rheumatoid arthritis," The Journal of Rheumatology, vol. 16, no. 19, pp. 154-157, 1989.

[37] K. Wingenfeld, D. Wagner, I. Schmidt, G. Meinlschmidt, D. H. Hellhammer, and C. Heim, "The low-dose dexamethasone suppression test in fibromyalgia," Journal of Psychosomatic Research, vol. 62, no. 1, pp. 85-91, 2007.

[38] J. A. Macedo, J. Hesse, J. D. Turner, J. Meyer, D. H. Hellhammer, and C. P. Muller, "Glucocorticoid sensitivity in fibromyalgia patients: decreased expression of corticosteroid receptors and glucocorticoid-induced leucine zipper," Psychoneuroendocrino$\log y$, vol. 33, no. 6, pp. 799-809, 2008.

[39] K. Wingenfeld, C. Heim, I. Schmidt, D. Wagner, G. Meinlschmidt, and D. H. Hellhammer, "HPA axis reactivity and lymphocyte glucocorticoid sensitivity in fibromyalgia syndrome and chronic pelvic pain," Psychosomatic Medicine, vol. 70, no. 1, pp. 65-72, 2008.

[40] S. Sudhaus, B. Fricke, S. Schneider et al., "The cortisol awakening response in patients with acute and chronic low back pain: relations with psychological risk factors of pain chronicity," Schmerz, vol. 21, no. 3, pp. 202-211, 2007.

[41] V. Sveinsdottir, H. R. Eriksen, H. Ursin, Å. M. Hansen, and A. Harris, "Cortisol, health, and coping in patients with nonspecific low back pain," Applied Psychophysiology Biofeedback, vol. 41, no. 1, pp. 9-16, 2016.

[42] K. B. Jo, Y. J. Lee, I. G. Lee, S. C. Lee, J. Y. Park, and R. S. Ahn, "Association of pain intensity, pain-related disability, and depression with hypothalamus-pituitary-adrenal axis function in female patients with chronic temporomandibular disorders," Psychoneuroendocrinology, vol. 69, pp. 106-115, 2016.

[43] A. V. Apkarian, M. C. Bushnell, R.-D. Treede, and J.-K. Zubieta, "Human brain mechanisms of pain perception and regulation in health and disease," European Journal of Pain, vol. 9, no. 4, pp. 463-484, 2005.

[44] M. C. Bushnell, M. Čeko, and L. A. Low, "Cognitive and emotional control of pain and its disruption in chronic pain," Nature Reviews Neuroscience, vol. 14, no. 7, pp. 502-511, 2013.

[45] R. Peyron, B. Laurent, and L. García-Larrea, "Functional imaging of brain responses to pain. A review and meta-analysis (2000)," Clinical Neurophysiology, vol. 30, no. 5, pp. 263-288, 2000.

[46] S. W. G. Derbyshire, A. K. P. Jones, F. Gyulai, S. Clark, D. Townsend, and L. L. Firestone, "Pain processing during three levels of noxious stimulation produces differential patterns of central activity," Pain, vol. 73, no. 3, pp. 431-445, 1997.

[47] N. Maleki, L. Becerra, J. Brawn, B. McEwen, R. Burstein, and D. Borsook, "Common hippocampal structural and functional changes in migraine," Brain Structure \& Function, vol. 218, no. 4, pp. 903-912, 2013.

[48] D. A. Matre, L. Hernandez-Garcia, T. D. Tran, and K. L. Casey, "'First pain' in humans: convergent and specific forebrain responses," Molecular Pain, vol. 6, no. 1, article 81, 2010.

[49] A. R. Segerdahl, M. Mezue, T. W. Okell, J. T. Farrar, and I. Tracey, "The dorsal posterior insula subserves a fundamental role in human pain," Nature Neuroscience, vol. 18, no. 4, pp. 499-500, 2015.

[50] T. D. Tran, H. Wang, A. Tandon, L. Hernandez-Garcia, and K. L. Casey, "Temporal summation of heat pain in humans: evidence supporting thalamocortical modulation," Pain, vol. 150, no. 1, pp. 93-102, 2010.

[51] C. H. Wilder-Smith, D. Schindler, K. Lovblad, S. M. Redmond, and A. Nirkko, "Brain functional magnetic resonance imaging of rectal pain and activation of endogenous inhibitory mechanisms in irritable bowel syndrome patient subgroups and healthy controls," Gut, vol. 53, no. 11, pp. 1595-1601, 2004.

[52] C. Berna, S. Leknes, E. A. Holmes, R. R. Edwards, G. M. Goodwin, and I. Tracey, "Induction of depressed mood disrupts emotion regulation neurocircuitry and enhances pain unpleasantness," Biological Psychiatry, vol. 67, no. 11, pp. 1083-1090, 2010.

[53] K. Bornhövd, M. Quante, V. Glauche, B. Bromm, C. Weiller, and C. Büchel, "Painful stimuli evoke different stimulus-response functions in the amygdala, prefrontal, insula and somatosensory cortex: a single-trial fMRI study," Brain, vol. 125, no. 6, pp. 1326-1336, 2002.

[54] U. Bingel, M. Quante, R. Knab, B. Bromm, C. Weiller, and C. Büchel, "Subcortical structures involved in pain processing: evidence from single-trial fMRI," Pain, vol. 99, no. 1-2, pp. 313321, 2002.

[55] M. Ziv, R. Tomer, R. Defrin, and T. Hendler, "Individual sensitivity to pain expectancy is related to differential activation of the hippocampus and amygdala," Human Brain Mapping, vol. 31, no. 2, pp. 326-338, 2010.

[56] A. Ploghaus, I. Tracey, J. S. Gati et al., "Dissociating pain from its anticipation in the human brain," Science, vol. 284, no. 5422, pp. 1979-1981, 1999.

[57] I. A. Strigo, A. N. Simmons, S. C. Matthews, A. D. Craig, and M. P. Paulus, "Increased affective bias revealed using experimental graded heat stimuli in young depressed adults: evidence of "emotional allodynia"," Psychosomatic Medicine, vol. 70, no. 3, pp. 338-344, 2008.

[58] S. Benson, L. Rebernik, A. Wegner et al., "Neural circuitry mediating inflammation-induced central pain amplification in human experimental endotoxemia," Brain, Behavior, \& Immunity, vol. 48, no. 1, pp. 222-231, 2015.

[59] J. C. Choi, J. Kim, E. Kang et al., "Step-down vs. step-up noxious stimulation: differential effects on pain perception and patterns of brain activation," Acta Anaesthesiologica Scandinavica, vol. 60, no. 1, pp. 117-127, 2016.

[60] J. F. Bernard, G. F. Huang, and J. M. Besson, "Nucleus centralis of the amygdala and the globus pallidus ventralis: electrophysiological evidence for an involvement in pain processes," Journal of Neurophysiology, vol. 68, no. 2, pp. 551-569, 1992.

[61] W. Li and V. Neugebauer, "Differential roles of mGluR1 and mGluR5 in brief and prolonged nociceptive processing in central amygdala neurons," Journal of Neurophysiology, vol. 91, no. 1, pp. 13-24, 2004.

[62] V. Neugebauer and W. Li, "Differential sensitization of amygdala neurons to afferent inputs in a model of arthritic pain," Journal of Neurophysiology, vol. 89, no. 2, pp. 716-727, 2003.

[63] R. Zhang, M. Tomida, Y. Katayama, and Y. Kawakami, "Response durations encode nociceptive stimulus intensity in the rat medial prefrontal cortex," Neuroscience, vol. 125, no. 3, pp. 777-785, 2004.

[64] S. Khanna, "Dorsal hippocampus field CA1 pyramidal cell responses to a persistent versus an acute nociceptive stimulus and 
their septal modulation," Neuroscience, vol. 77, no. 3, pp. 713721, 1997.

[65] S. Khanna and J. G. Sinclair, "Noxious stimuli produce prolonged changes in the CA1 region of the rat hippocampus," Pain, vol. 39, no. 3, pp. 337-343, 1989.

[66] Z. Wang, S. Bradesi, J.-M. I. Maarek et al., "Regional brain activation in conscious, nonrestrained rats in response to noxious visceral stimulation," Pain, vol. 138, no. 1, pp. 233-243, 2008.

[67] T. Hayashi, M. Miyata, T. Nagata, Y. Izawa, and Y. Kawakami, "Intracerebroventricular fluvoxamine administration inhibited pain behavior but increased Fos expression in affective pain pathways," Pharmacology Biochemistry \& Behavior, vol. 91, no. 3, pp. 441-446, 2009.

[68] S. Khanna, L. S. Chang, F. Jiang, and H. C. Koh, "Nociceptiondriven decreased induction of Fos protein in ventral hippocampus field CA1 of the rat," Brain Research, vol. 1004, no. 1-2, pp. 167-176, 2004.

[69] T. Nakagawa, A. Katsuya, S. Tanimoto et al., "Differential patterns of c-fos mRNA expression in the amygdaloid nuclei induced by chemical somatic and visceral noxious stimuli in rats," Neuroscience Letters, vol. 344, no. 3, pp. 197-200, 2003.

[70] M. Lehner, E. Taracha, A. Skórzewska et al., "Behavioral, immunocytochemical and biochemical studies in rats differing in their sensitivity to pain," Behavioural Brain Research, vol. 171, no. 2, pp. 189-198, 2006.

[71] A. V. Apkarian, Y. Sosa, S. Sonty et al., "Chronic back pain is associated with decreased prefrontal and thalamic gray matter density," The Journal of Neuroscience, vol. 24, no. 46, pp. 1041010415, 2004.

[72] A. May, "Chronic pain may change the structure of the brain," Pain, vol. 137, no. 1, pp. 7-15, 2008.

[73] R. F. Smallwood, A. R. Laird, A. E. Ramage et al., "Structural brain anomalies and chronic pain: a quantitative meta-analysis of gray matter volume," Journal of Pain, vol. 14, no. 7, pp. 663675, 2013.

[74] M. Burgmer, M. Gaubitz, C. Konrad et al., "Decreased gray matter volumes in the cingulo-frontal cortex and the amygdala in patients with fibromyalgia," Psychosomatic Medicine, vol. 71, no. 5, pp. 566-573, 2009.

[75] J. S. Labus, I. D. Dinov, Z. Jiang et al., "Irritable bowel syndrome in female patients is associated with alterations in structural brain networks," Pain, vol. 155, no. 1, pp. 137-149, 2014.

[76] C. P. Mao and H. J. Yang, "Smaller amygdala volumes in patients with chronic low back pain compared with healthy control individuals," Journal of Pain, vol. 16, no. 12, pp. 1366-1376, 2015.

[77] R. Rodriguez-Raecke, A. Niemeier, K. Ihle, W. Ruether, and A. May, "Brain gray matter decrease in chronic pain is the consequence and not the cause of pain," The Journal of Neuroscience, vol. 29, no. 44, pp. 13746-13750, 2009.

[78] W. Valfrè, I. Rainero, M. Bergui, and L. Pinessi, "Voxel-based morphometry reveals gray matter abnormalities in migraine," Headache, vol. 48, no. 1, pp. 109-117, 2008.

[79] U. Blankstein, J. Chen, N. E. Diamant, and K. D. Davis, "Altered brain structure in irritable bowel syndrome: potential contributions of pre-existing and disease-driven factors," Gastroenterology, vol. 138, no. 5, pp. 1783-1789, 2010.

[80] P. Y. Geha, M. N. Baliki, R. N. Harden, W. R. Bauer, T. B. Parrish, and A. V. Apkarian, "The brain in chronic CRPS pain: abnormal gray-white matter interactions in emotional and autonomic regions," Neuron, vol. 60, no. 4, pp. 570-581, 2008.
[81] T. Schmidt-Wilcke, E. Leinisch, A. Straube et al., "Gray matter decrease in patients with chronic tension type headache," Neurology, vol. 65, no. 9, pp. 1483-1486, 2005.

[82] J. S. Labus, C. S. Hubbard, J. Bueller et al., "Impaired emotional learning and involvement of the corticotropin-releasing factor signaling system in patients with irritable bowel syndrome," Gastroenterology, vol. 145, no. 6, pp. 1253-1261.e3, 2013.

[83] A. A. Mutso, D. Radzicki, M. N. Baliki et al., "Abnormalities in hippocampal functioning with persistent pain," The Journal of Neuroscience, vol. 32, no. 17, pp. 5747-5756, 2012.

[84] A. V. Apkarian, P. S. Thomas, B. R. Krauss, and N. M. Szeverenyi, "Prefrontal cortical hyperactivity in patients with sympathetically mediated chronic pain," Neuroscience Letters, vol. 311, no. 3, pp. 193-197, 2001.

[85] L. E. Simons, E. A. Moulton, C. Linnman, E. Carpino, L. Becerra, and D. Borsook, "The human amygdala and pain: evidence from neuroimaging," Human Brain Mapping, vol. 35, no. 2, pp. 527-538, 2014.

[86] M. N. Baliki, D. R. Chialvo, P. Y. Geha et al., "Chronic pain and the emotional brain: specific brain activity associated with spontaneous fluctuations of intensity of chronic back pain," The Journal of Neuroscience, vol. 26, no. 47, pp. 12165-12173, 2006.

[87] P. Schweinhardt, C. Glynn, J. Brooks et al., "An fMRI study of cerebral processing of brush-evoked allodynia in neuropathic pain patients," NeuroImage, vol. 32, no. 1, pp. 256-265, 2006.

[88] A. A. Mutso, B. Petre, L. Huang et al., "Reorganization of hippocampal functional connectivity with transition to chronic back pain," Journal of Neurophysiology, vol. 111, no. 5, pp. 1065-1076, 2014.

[89] D. Niddam, S. Lee, Y. Su, and R. Chan, "Brain structural changes in patients with chronic myofascial pain," European Journal of Pain, 2016.

[90] R. Ikeda, Y. Takahashi, K. Inoue, and F. Kato, "NMDA receptorindependent synaptic plasticity in the central amygdala in the rat model of neuropathic pain," Pain, vol. 127, no. 1-2, pp. 161$172,2007$.

[91] M. Tajerian, D. Leu, Y. Zou et al., "Brain neuroplastic changes accompany anxiety and memory deficits in a model of complex regional pain syndrome," Anesthesiology, vol. 121, no. 4, pp. 852865, 2014.

[92] L. Gonçalves, R. Silva, F. Pinto-Ribeiro et al., "Neuropathic pain is associated with depressive behaviour and induces neuroplasticity in the amygdala of the rat," Experimental Neurology, vol. 213, no. 1, pp. 48-56, 2008.

[93] T. Kiritoshi and V. Neugebauer, "Group II mGluRs modulate baseline and arthritis pain-related synaptic transmission in the rat medial prefrontal cortex," Neuropharmacology, vol. 95, pp. 388-394, 2015.

[94] A. E. Metz, H.-J. Yau, M. V. Centeno, A. V. Apkarian, and M. Martina, "Morphological and functional reorganization of rat medial prefrontal cortex in neuropathic pain," Proceedings of the National Academy of Sciences of the United States of America, vol. 106, no. 7, pp. 2423-2428, 2009.

[95] P. Schweinhardt, D. A. Seminowicz, E. Jaeger, G. H. Duncan, and M. C. Bushnell, "The anatomy of the mesolimbic reward System: a link between personality and the placebo analgesic response," Journal of Neuroscience, vol. 29, no. 15, pp. 4882-4887, 2009.

[96] M. Terada, N. Kuzumaki, N. Hareyama et al., "Suppression of enriched environment-induced neurogenesis in a rodent model of neuropathic pain," Neuroscience Letters, vol. 440, no. 3, pp. 314-318, 2008. 
[97] W. B. Hoover and R. P. Vertes, "Anatomical analysis of afferent projections to the medial prefrontal cortex in the rat," Brain Structure \& Function, vol. 212, no. 2, pp. 149-179, 2007.

[98] G. Ji and V. Neugebauer, "CB1 augments mGluR5 function in medial prefrontal cortical neurons to inhibit amygdala hyperactivity in an arthritis pain model," European Journal of Neuroscience, vol. 39, no. 3, pp. 455-466, 2014.

[99] G. Ji, H. Sun, Y. Fu et al., "Cognitive impairment in pain through amygdala-driven prefrontal cortical deactivation," The Journal of Neuroscience, vol. 30, no. 15, pp. 5451-5464, 2010.

[100] H. Cardoso-Cruz, D. Lima, and V. Galhardo, "Impaired spatial memory performance in a rat model of neuropathic pain is associated with reduced hippocampus-prefrontal cortex connectivity," The Journal of Neuroscience, vol. 33, no. 6, pp. 24652480, 2013.

[101] Y. M. Ulrich-Lai, W. Xie, J. T. A. Meij, C. M. Dolgas, L. Yu, and J. P. Herman, "Limbic and HPA axis function in an animal model of chronic neuropathic pain," Physiology \& Behavior, vol. 88, no. 1-2, pp. 67-76, 2006.

[102] S. J. Lupien, B. S. McEwen, M. R. Gunnar, and C. Heim, "Effects of stress throughout the lifespan on the brain, behaviour and cognition," Nature Reviews Neuroscience, vol. 10, no. 6, pp. 434445, 2009.

[103] R. M. Sapolsky, L. C. Krey, and B. S. Mcewen, "The neuroendocrinology of stress and aging: the glucocorticoid cascade hypothesis," Endocrine Reviews, vol. 7, no. 3, pp. 284-301, 1986.

[104] M. W. Gilbertson, M. E. Shenton, A. Ciszewski et al., "Smaller hippocampal volume predicts pathologic vulnerability to psychological trauma," Nature Neuroscience, vol. 5, no. 11, pp. 12421247, 2002.

[105] D. M. Lyons, C. Yang, A. M. Sawyer-Glover, M. E. Moseley, and A. F. Schatzberg, "Early life stress and inherited variation in monkey hippocampal volumes," Archives of General Psychiatry, vol. 58, no. 12, pp. 1145-1151, 2001.

[106] I. N. Karatsoreos and B. S. McEwen, "Psychobiological allostasis: resistance, resilience and vulnerability," Trends in Cognitive Sciences, vol. 15, no. 12, pp. 576-584, 2011.

[107] R. M. Sapolsky, H. Uno, C. S. Rebert, and C. E. Finch, "Hippocampal damage associated with prolonged glucocorticoid exposure in primates," Journal of Neuroscience, vol. 10, no. 9, pp. 2897-2902, 1990.

[108] L. E. Simons, I. Elman, and D. Borsook, "Psychological processing in chronic pain: a neural systems approach," Neuroscience and Biobehavioral Reviews, vol. 39, pp. 61-78, 2014.

[109] D. P. Hibar, J. L. Stein, M. E. Renteria et al., "Common genetic variants influence human subcortical brain structures," Nature, vol. 520, no. 7546, pp. 224-229, 2015.

[110] S. J. Lupien, F. Maheu, M. Tu, A. Fiocco, and T. E. Schramek, "The effects of stress and stress hormones on human cognition: implications for the field of brain and cognition," Brain and Cognition, vol. 65, no. 3, pp. 209-237, 2007.

[111] M. N. Baliki and A. V. Apkarian, "Nociception, pain, negative moods, and behavior selection," Neuron, vol. 87, no. 3, pp. 474491, 2015. 

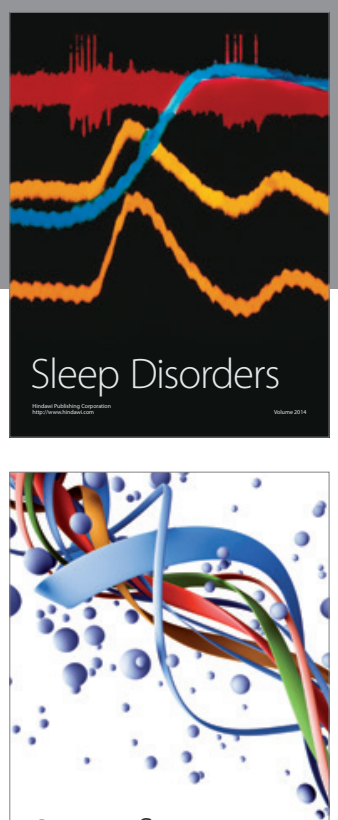

Scientifica
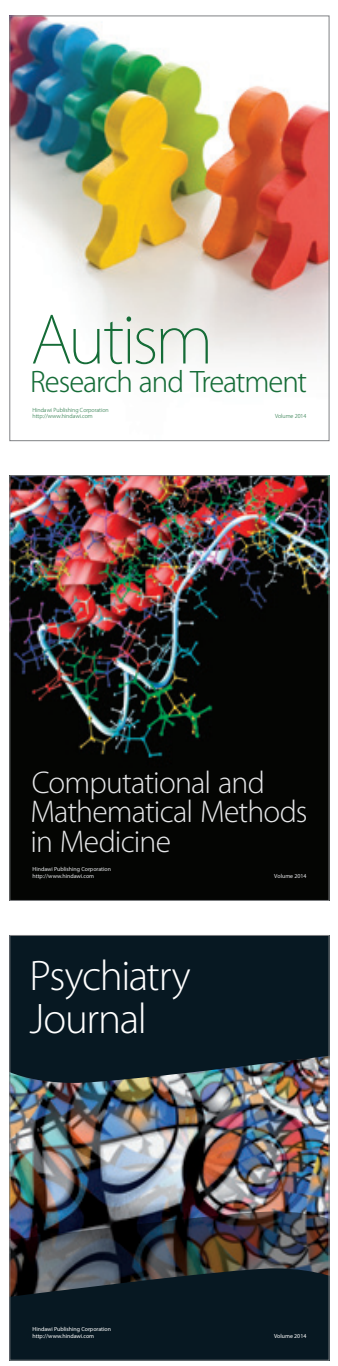
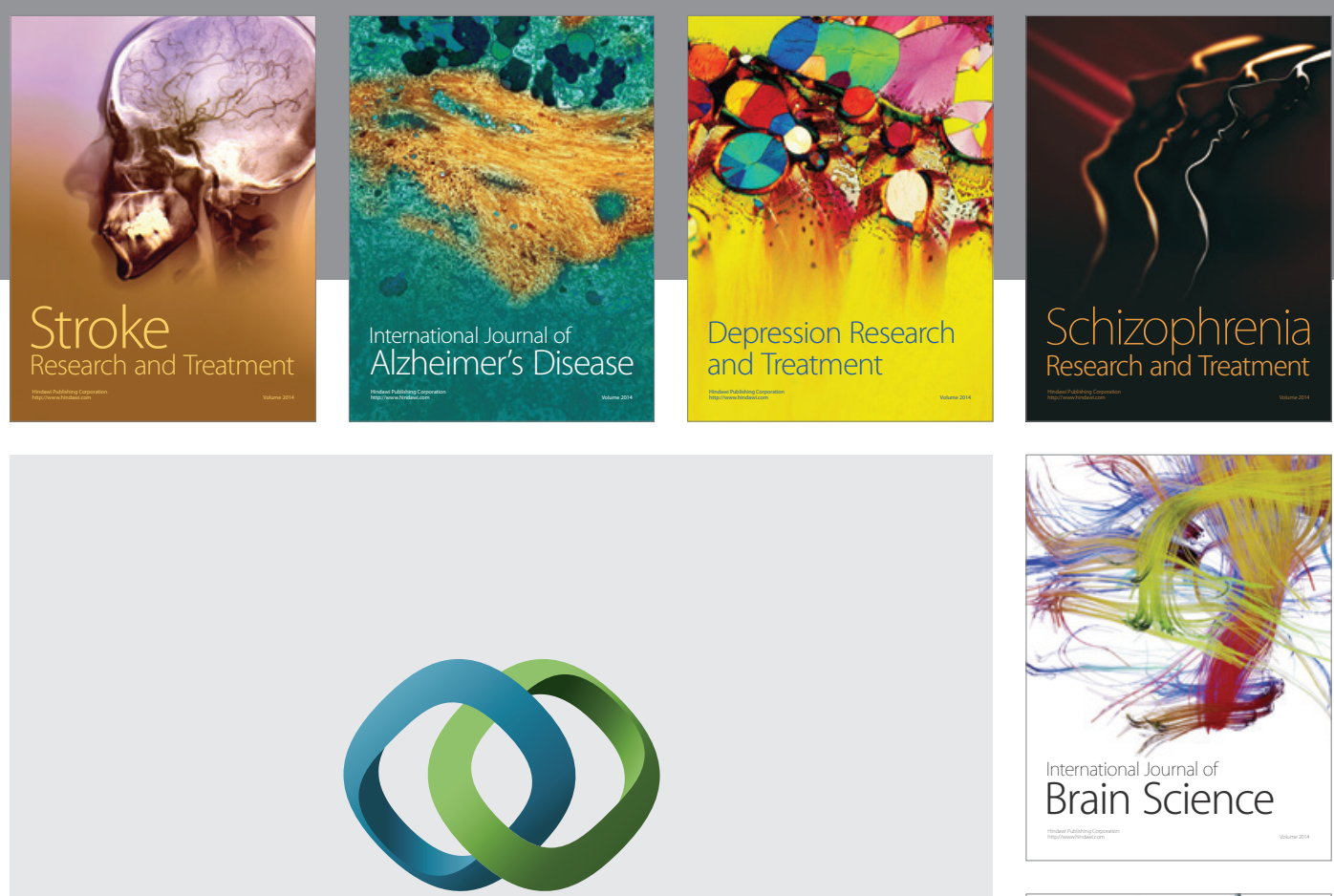

\section{Hindawi}

Submit your manuscripts at

http://www.hindawi.com
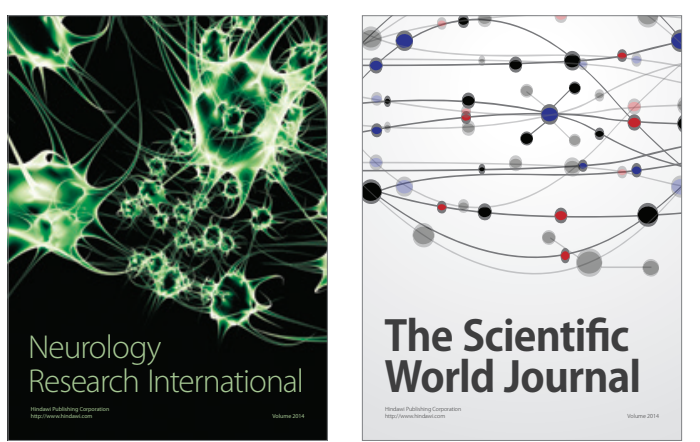

The Scientific World Journal

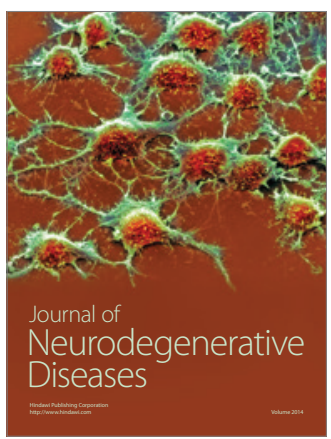

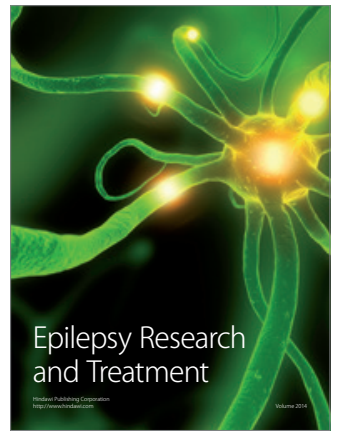

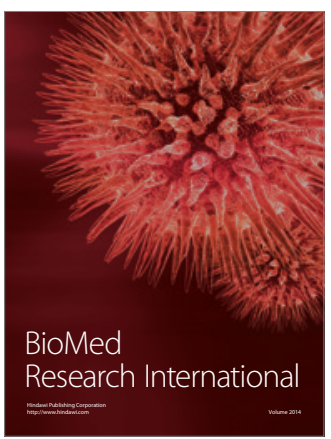

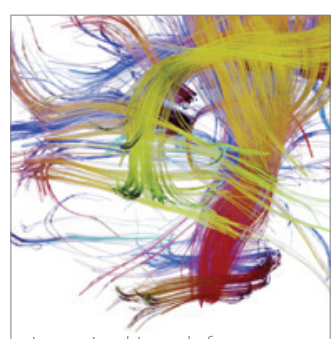

Brain Science

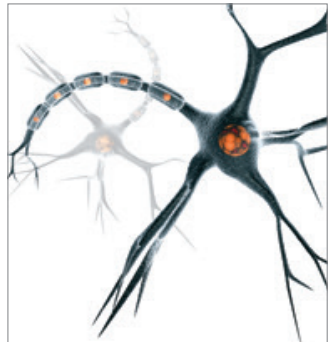

Neural Plasticity
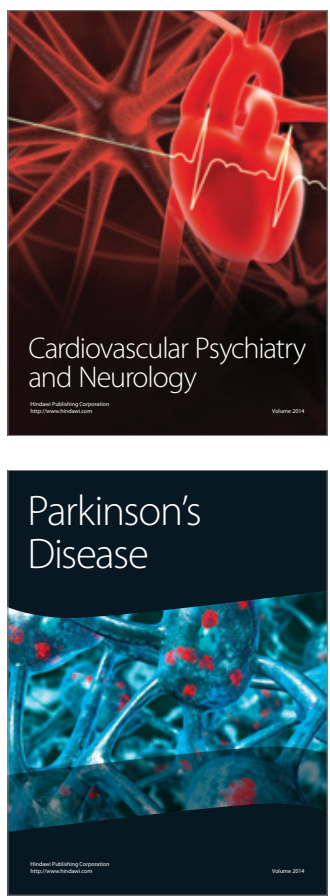